\title{
Compact Kähler threefolds with non-nef canonical bundle and symplectic geometry
}

\author{
HSUEH-YUNG LIN
}

We show that the nefness of the canonical bundle of compact Kähler threefolds is invariant under deformed symplectic diffeomorphisms.

\section{Introduction}

Consider a compact Kähler manifold $(X, \omega)$ and its canonical bundle $K_{X}$. We say that $K_{X}$ is algebraically nef if its degree is non-negative on every curve $C$ on $X$. We shall briefly call it nef ${ }^{1}$ throughout this note by abuse of language. Let $\left(Y, \omega^{\prime}\right)$ be another compact Kähler manifold. A deformed symplectic diffeomorphism $\phi: X \rightarrow Y$ is a diffeomorphism such that the symplectic form $\phi^{*} \omega^{\prime}$ is in the same deformation class of symplectic forms as $\omega$. If such a diffeomorphism $\phi$ exists, we say that $X$ and $Y$ are symplectically equivalent.

A Kähler manifold equipped with its Kähler form is also a symplectic manifold, and furthermore, the set of Kähler forms is connected and thus determines a deformation class of symplectic forms. Hence, it is natural to ask which properties coming from algebraic or Kähler geometry depend only on the symplectic deformation equivalence class. This kind of questions was first studied by Ruan $[11,12]$ by showing that the nefness of the canonical bundle of smooth projective surfaces or threefolds is invariant under deformed symplectic diffeomorphisms. In the same direction, Kollár [7] and Ruan [13] showed that uniruledness of Kähler manifolds is a symplectic invariant. The same holds true for rational connectedness of smooth projective varieties of dimension up to four $[15,16,18]$.

\footnotetext{
${ }^{1}$ Usually, a line bundle $L$ on a Kähler manifold $(X, \omega)$ is called nef if for every $\varepsilon>$ 0 , there exists a Hermitian metric on $L$ with curvature $\Theta \geq-\varepsilon \omega$. This is stronger than algebraic nefness, but these two notions coincide when $X$ is a smooth projective variety or a compact Kähler threefold [6].
} 
The aim of this note is to prove the following Theorem, which generalizes Ruan's result:

Theorem 1.1. The algebraic nefness of the canonical bundle of compact Kähler threefolds is invariant under deformed symplectic diffeomorphisms: given two symplectically equivalent compact Kähler threefolds $X$ and $Y$, assume that $K_{X}$ is nef, then $K_{Y}$ is also nef.

Let $X$ be a compact Kähler manifold. Set $N_{1}(X)_{\mathbb{R}}^{2}$ the real vector space of 1-cycles modulo numerical equivalence. Inside $N_{1}(X)_{\mathbb{R}}$ sits the effective cone

$$
\mathrm{NE}(X):=\left\{\sum_{\text {finite }} a_{i}\left[C_{i}\right] \mid a_{i}>0, C_{i} \text { irreducible curves of } X\right\} \subset N_{1}(X)_{\mathbb{R}}
$$

the set of classes of effective 1-cycles. We denote by $\overline{\mathrm{NE}}(X)$ the closure of $\mathrm{NE}(X)$ in $N_{1}(X)_{\mathbb{R}}$.

More generally, given a symplectic manifold $(M, \omega)$ and a $\omega$-tamed almost complex structure $J$ on $M$, we define

$$
\mathrm{NE}(M)_{\omega, J}:=\left\{\sum_{\text {finite }} a_{i}\left[C_{i}\right] \mid a_{i}>0, C_{i} J \text {-holomorphic curves of } M\right\} \subset H_{2}(M, \mathbb{R}) .
$$

In [11], Ruan defined the deformed symplectic effective cone as the intersection for all symplectic forms $\omega^{\prime}$ which can deform to $\omega$ and all $\omega^{\prime}$-tamed almost complex structures $J$

$$
\operatorname{DNE}(M):=\bigcap_{\omega^{\prime}} \bigcap_{J} \mathrm{NE}(M)_{\omega, J}
$$

In the same paper, he stated the following main criterion:

Theorem 1.2. Suppose that $[C]$ is extremal ${ }^{3}$ in $\overline{\mathrm{NE}}(M)_{\omega^{\prime}, J}$ for some $\omega^{\prime}$ and $J$. If the Gromov-Witten invariant $\mathrm{GW}_{[C]}$ is non-zero, then $[C]$ is extremal in $\overline{\mathrm{DNE}}(M)$.

\footnotetext{
${ }^{2}$ By Hodge theory, this coincides with the set of real Hodge classes $H^{1,1}(X, \mathbb{C}) \cap$ $H^{2}(X, \mathbb{R})$.

${ }^{3}$ An element $x$ in a closed cone $C$ is called extremal if whenever $x=y+z$ for $y, z \in C$, then $y$ and $z$ are multiples of $x$.
} 
When $X$ is a smooth projective threefold, Mori gave a classification of the extremal rays in

$$
\overline{\mathrm{NE}}(X)_{K_{X}<0}:=\overline{\left\{[C] \in \mathrm{NE}(X) \mid\left(K_{X}, C\right)<0\right\}}
$$

and showed that they are all generated by rational curves. We define the minimal homology class in an extremal ray $R$ the class of a rational curve $[C] \in R$ such that $\left(-K_{X}, C\right)$ is minimal. Ruan used his main criterion to prove that extremal curves do not disappear under symplectic diffeomorphism for projective threefolds by verifying that a minimal homology class $[C]$ in each extremal ray of $\overline{\mathrm{NE}}(X)_{K_{X}<0}$ has a non-zero genus zero GromovWitten invariant $\mathrm{GW}_{0,[C]}$. Since the canonical class is also a symplectic invariant, i.e., $\phi^{*} c_{1}\left(K_{Y}\right)=c_{1}\left(K_{X}\right)$ (indeed, $c_{1}\left(K_{X}\right)$ is determined by any $\omega$-tamed almost complex structure which form a connected set) and since genus zero Gromov-Witten invariants can be calculated by doing intersection theory on the moduli space of rational curves [8] [14], we conclude that there exists a rational curve $C^{\prime} \subset Y$ such that $\phi^{*}\left[C^{\prime}\right]=[C]$ and $\left(K_{Y}, C^{\prime}\right)=\left(\phi^{*} K_{Y}, \phi^{*}\left[C^{\prime}\right]\right)=\left(K_{X}, C\right)<0$.

Back to the Kähler case, since the projective case was verified by Ruan, it suffices to prove Theorem 1.1 for non-algebraic compact Kähler threefolds. Recently, Höring and Peternell generalized Mori's cone Theorem for compact Kähler threefolds [6].

Theorem 1.3 (Höring, Peternell). Let $X$ be a compact Kähler threefold. There exists a countable set $\left(C_{i}\right)_{i \in I}$ of rational curves on $X$ such that

$$
0<-K_{X} \cdot C_{i} \leq \operatorname{dim}(X)+1
$$

and

$$
\overline{\mathrm{NE}}(X)=\overline{\mathrm{NE}}(X)_{K_{X} \geq 0}+\sum_{i \in I} \mathbb{R}^{+}\left[C_{i}\right]
$$

where the $\mathbb{R}^{+}\left[C_{i}\right]$ are all the distinct extremal rays of $\overline{\mathrm{NE}}(X)$ that meet $N_{1}(X)_{K_{X}<0}$. These rays are locally discrete in that half-space.

This Theorem together with the classification list of non-splitting families of rational curves on Kähler threefolds given by Campana and Peternell [3] allow us to follow Ruan's method to prove Theorem 1.1 in the Kähler context.

This note is organized as follows. In Section 2, we exhibit the classification list of non-splitting families of rational curves on Kähler threefolds. 
In Section 3, we recall some elementary properties of Gromov-Witten invariants which we will use. Finally, we prove Theorem 1.1 in the last section.

If $Z$ is an analytic (sub)space, $[Z]$ will denote interchangeably its fundamental homology class and its Poincaré dual cohomology class throughout this note.

\section{Classification of non-splitting families of rational curves}

Let $X$ be a compact Kähler threefold. A family of rational curves $\left(C_{t}\right)_{t \in T}$ in $X$ is called non-splitting, if $T$ is compact and irreducible and every curve $C_{t}$ is irreducible. Every extremal rational curve $C$ such that $\left(K_{X}, C\right)<0$ determines a non-splitting family of rational curves $\left(C_{t}\right)_{t \in T}$ [3], and dim $T=-\left(K_{X}, C\right)$ by definition. One can classify these families according to $-\left(K_{X}, C\right) \in\{1,2,3,4\}$ and this was done by Campana and Peternell [3]. Since we are mainly interested in non-algebraic compact threefolds, we shall exclude projective varieties from the classification list.

Theorem 2.1. Let $X$ be a non-algebraic compact Kähler threefold and $\left(C_{t}\right)$ a non-splitting family of rational curves. Then either $\left(K_{X}, C_{t}\right)=-2$ or -1 . Moreover,

(1) If $\left(K_{X}, C_{t}\right)=-2$, then

(a) if $C_{t}$ fills up a surface $S \subset X$, then $S \simeq \mathbb{P}^{2}$ with normal bundle $N_{S / X}=\mathscr{O}(-1)$ and $\left(C_{t}\right)$ is the family of lines;

(b) if $C_{t}$ fills up $X$, then $X$ is a $\mathbb{P}^{1}$-bundle over a surface, the $C_{t}$ being fibers.

(2) If $\left(K_{X}, C_{t}\right)=-1$, then $C_{t}$ fills up a surface $S \subset X$.

(a) If $S$ is normal, then one of the following holds:

(i) $S \simeq \mathbb{P}^{2}$ with $N_{S / X}=\mathscr{O}(-2)$ and $\left(C_{t}\right)$ is the family of lines;

(ii) $S \simeq \mathbb{P}^{1} \times \mathbb{P}^{1}$ with $N_{S / X}=\mathscr{O}(-1) \times \mathscr{O}(-1)$ and the $C_{t}$ are lines in $S$;

(iii) $S$ is a quadric cone with $N_{S / X}=\mathscr{O}(-1)$;

(iv) $S$ is a ruled surface over a smooth curve $B$, the $C_{t}$ being fibers of $S \rightarrow B$, and $X$ is the blow-up of a smooth threefold along $B$.

(b) If $S$ is non-normal, then $\kappa(X)<0$ and $N_{S / X}=\mathscr{O}_{S}$. The normalization $\tilde{S}$ of $S$ is either $\mathbb{P}^{2}$ or a ruled surface $\pi: \tilde{S} \rightarrow B$. In the formal case, the strict transform $\tilde{C}_{t}$ of $C_{t}$ under $\nu$ is a line in $\mathbb{P}^{2}$. In the latter case, the ruling $\pi$ of $\tilde{S}$ can be chosen so that $\tilde{C}_{t}$ is a fiber of $\pi$. 


\section{Preliminaries on Gromov-Witten invariants}

Let $A \in H_{2}(X, \mathbb{Z})$. We denote by $\overline{\mathscr{M}}_{A, n}(X)$ (or $\overline{\mathscr{M}}_{A, n}$ ) the moduli space of stable maps from curves of genus 0 to $X$ with $n$ marked points, whose homology class of its image is equal to $A$. When there is no marked point, this moduli space is simply denoted by $\overline{\mathscr{M}}_{A}$. It is a proper Deligne-Mumford stack. Since $X$ is a complex threefold, we recall that the expected (or virtual) dimension of $\overline{\mathscr{M}}_{A, n}$

$$
d_{\exp }=n-\int_{A} c_{1}\left(K_{X}\right)
$$

and $\overline{\mathscr{M}}_{A, n}$ carries a virtual fundamental class of expected dimension $2 d_{\exp }$

$$
\left[\overline{\mathscr{M}}_{A, n}\right]^{\mathrm{vir}} \in H_{2 d_{\text {exp }}}\left(\overline{\mathscr{M}}_{A, n}, \mathbb{Q}\right) \text {. }
$$

Gromov-Witten invariants are defined by capping the cohomology classes against the virtual fundamental class of the space of stable maps. More precisely, given cohomology classes $A_{1}, \ldots, A_{n}$ in $H^{*}(X, \mathbb{Q})$, the corresponding genus zero Gromov-Witten invariant is defined by:

$$
G W_{0, A}\left(A_{1}, \ldots, A_{n}\right):=\int_{\left[\overline{\mathscr{M}}_{A, n}\right]^{\mathrm{vir}}} e_{1}^{*}\left(A_{1}\right) \smile \cdots \smile e_{n}^{*}\left(A_{n}\right),
$$

where $e_{i}$ denotes the evaluation map with respect to the $i$ th marked point

$$
\begin{aligned}
e_{i}: \overline{\mathscr{M}}_{A, n} & \longrightarrow X \\
\left(\Sigma ; p_{1}, \ldots, p_{n} ; f\right) & \longmapsto f\left(p_{i}\right) .
\end{aligned}
$$

When there is no obstruction on $\overline{\mathscr{M}}_{A, n}$, virtual fundamental class coincides with ordinary fundamental class. For instance, this happens when $H^{1}\left(C, f^{*} T X\right)=0$ for all stable maps $f: C \rightarrow X[4]$. When $\overline{\mathscr{M}}_{A, n}$ is nonsingular, the canonical obstruction sheaf $\mathscr{T}^{2}$ is a vector bundle, so the virtual fundamental class is the Euler class of $\mathscr{T}^{2}$ :

$$
\left[\overline{\mathscr{M}}_{A, n}\right]^{\mathrm{vir}}=e\left(\mathscr{T}^{2}\right) \cap\left[\overline{\mathscr{M}}_{A, n}\right]
$$

see [5] for more details.

For each embedded curve $f: C \rightarrow X$, the pushforward by $f$ of the fundamental class of $C$ and its Poincaré dual in $H^{2}(X, \mathbb{Z})$ are all denoted by $[C]$ in this note. 
Remark 3.1. Theorem 1.1 is true for compact Kähler surfaces for simple reasons. Let $X$ and $Y$ be compact Kähler surfaces. If $K_{X}$ is not nef, then either $X$ contains a $(-1)$-curve $C$, or $\kappa(X)<0$. If $f: C \rightarrow X$ is an embedded $(-1)$-curve, then $H^{1}\left(C, f^{*} T X\right)=H^{1}\left(\mathbb{P}^{1}, \mathscr{O}(-1)\right)=0$, so there is no obstruction on $\overline{\mathscr{M}}_{[C]}$. The moduli space is zero-dimensional, and $\mathrm{GW}_{[C]}=1$. If $\kappa(X)<0$, then by classification of minimal compact Kähler surfaces, $X$ is uniruled. Since uniruledness is preserved by deformed symplectic diffeomorphism[7, 13], $Y$ is also uniruled. In either case, $K_{Y}$ is not nef.

\section{Non-nef canonical bundle and symplectic geometry}

This section is devoted to the proof of Theorem 1.1.

Proof of Theorem 1.1. Suppose $K_{X}$ is not nef, and $C$ is an extremal rational curve such that $\left(K_{X}, C\right)<0$ whose existence is given by Höring-Peternell's cone Theorem 1.3. The case where $X$ is projective was treated in [13]. As before, we thus assume that $X$ is non-algebraic. The curve $C$ determines a non-splitting family of rational curves $\left(C_{t}\right)$ which is classified in Theorem 2.1. Since the canonical class $c_{1}\left(K_{X}\right)$ is a symplectic invariant, it suffices to show that some genus zero Gromov-Witten invariants $G W_{0,[C]}(\cdots)$ is non-zero for all cases listed in Theorem 2.1.

Lemma 4.1. For the cases (1.a), (1.b), (2.a.ii), (2.a.iii) and (2.a.iv), the moduli space $\overline{\mathscr{M}}_{[C]}$ is unobstructed. Furthermore, $\mathrm{GW}_{0,[C]} \neq 0$.

Proof. Since the normal bundles $N_{C \mid X}$ in the cases (1.a), (1.b), (2.a.ii), (2.a.iii) and (2.a.iv) are

$$
\mathscr{O}(1) \oplus \mathscr{O}(-1), \mathscr{O} \oplus \mathscr{O}, \mathscr{O} \oplus \mathscr{O}(-1), \mathscr{O} \oplus \mathscr{O}(-1) \text {, and } \mathscr{O} \oplus \mathscr{O}(-1),
$$

respectively, one has $H^{1}\left(C, f^{*} T_{X}\right)=H^{1}\left(C, N_{C \mid X}\right)=0$, so the moduli space $\overline{\mathscr{M}}_{[C]}$ in the cases above is unobstructed.

For (1.a), let

$$
e:=\left(e_{1}, e_{2}\right): \overline{\mathscr{M}}_{[C], 2} \rightarrow X \times X
$$

where $e_{i}$ is the evaluation map with respect to the $i$ th marked point. Let $S \simeq \mathbb{P}^{2} \subset X$ denote the image of $e: \overline{\mathscr{M}}_{[C], 1} \rightarrow X$, then 


$$
\begin{aligned}
\mathrm{GW}_{0,[C]}([C],[C]) & =\int_{\overline{\mathscr{M}}_{[C], 2}} e^{*}[C \times C]=[C \times C] \cdot[S \times S] \\
& =p_{1}^{*}([C] \cdot[S]) \cdot p_{2}^{*}([C] \cdot[S])=1,
\end{aligned}
$$

where $p_{i}$ is the projection $X \times X \rightarrow X$ onto the $i$ th component.

For $(1 . b)$, it is clear that $\mathrm{GW}_{0,[C]}([x])=1$ with $x \in X$. In the cases (2.a.ii), (2.a.iii) and (2.a.iv), one has $\mathrm{GW}_{0,[C]}([C])=[C] \cdot[S]=-1$ by the projection formula.

Remark 4.2. Another way to show that $\mathrm{GW}_{0,[C]}([C],[C])=1$ in the case (1.a) is by perturbing (non-algebraically) a line $l \subset S$ out of the surface $S$ to get two lines $l^{\prime}$ and $l^{\prime \prime}$ intersecting $S$ transversally and negatively in two different points. We refer to the proof of Proposition 5.6 (case "Type $E_{2}$ ") in [13] for the detail.

Remark 4.3. We can also consider Lemma 4.1 for the cases (1.b), (2.a.ii), (2.a.iii) and (2.a.iv) as a consequence of the following Lemma, which slightly generalizes Lemma 5.3 in [13] in the context of Kähler geometry:

Lemma 4.4. Suppose that the deformation of $C$ is unobstructed. Let $e$ : $\overline{\mathscr{M}}_{[C], 1} \rightarrow X$ be the evaluation map. If $\operatorname{dim} \overline{\mathscr{M}}_{[C]} \leq 2$ and

$$
\operatorname{dim} e\left(\overline{\mathscr{M}}_{[C], 1}\right)=\operatorname{dim} \overline{\mathscr{M}}_{[C], 1},
$$

then $\mathrm{GW}_{0,[C]} \neq 0$.

Proof. Since $\operatorname{dim} e\left(\overline{\mathscr{M}}_{[C], 1}\right)=\operatorname{dim} \overline{\mathscr{M}}_{[C], 1}$, one has

$$
e^{*}\left[\overline{\mathscr{M}}_{[C], 1}\right]=k\left[e\left(\overline{\mathscr{M}}_{[C], 1}\right)\right]
$$

where $k=\operatorname{deg} e \neq 0$.

If $\operatorname{dim} \overline{\mathscr{M}}_{[C]}=2$ and $x \in X$ is a point in general position, then

$$
\mathrm{GW}_{0,[C]}(x)=e^{*}[x] \smile\left[\overline{\mathscr{M}}_{[C], 1}\right]=k \neq 0
$$

by projection formula. 
If $\operatorname{dim} \overline{\mathscr{M}}_{[C]}=1$, again by projection formula, one has

$$
\mathrm{GW}_{0,[C]}(\alpha)=k \int_{e\left(\overline{\mathscr{M}}_{[C], 1}\right)} \alpha,
$$

for $\alpha \in H^{4}(X, \mathbb{Q})$. Since $H^{4}(X, \mathbb{Q})$ is dense in $H^{4}(X, \mathbb{R})$, we can choose $\alpha \in$ $H^{4}(X, \mathbb{Q})$ sufficiently close to $\omega \wedge \omega$, hence

$$
\mathrm{GW}_{0,[C]}(\alpha)=k \int_{e\left(\overline{\mathscr{M}}_{[C], 1}\right)} \alpha \neq 0
$$

For (2.a.i), one has $H^{1}\left(C, f^{*} T_{X}\right)=H^{1}\left(C, N_{C \mid X}\right)=\mathbb{C}$, so the deformation of $C$ is obstructed. Since $\overline{\mathscr{M}}_{[C]} \simeq G(2,3) \simeq \mathbb{P}^{2}$ is non-singular, the virtual fundamental class

$$
\left[\overline{\mathscr{M}}_{A}\right]^{\mathrm{vir}}=e\left(\mathscr{T}^{2}\right) \cap\left[\overline{\mathscr{M}}_{A}\right]
$$

where $e\left(\mathscr{T}^{2}\right)$ is the Euler class of the obstruction bundle $\mathscr{T}^{2}$ and was first computed by Ruan [11].

Lemma 4.5. $e\left(\mathscr{T}^{2}\right)=-\sigma_{1}$ where $\sigma_{1}$ is the Schubert cycle which represent all the lines in $\mathbb{P}^{2}$ passing through a point in general position. Moreover, $\mathrm{GW}_{0,[C]}([C])=[C] \cdot[S]=-2$.

Proof. cf. [11], Section 5.

It remains the case $(2 . b)$, where $C_{t}$ fills up a non-normal surface $S$. We denote by $\nu: \tilde{S} \rightarrow S$ the normalization of $S$.

Remark 4.6. Using the abundance conjecture, proven for non-simple Kähler threefolds by Peternell [10] and for all Kähler threefolds by Campana et al. [2], the remaining case can be settled easily. Indeed, threefolds in case (2.b) are uniruled since they have negative Kodaira dimension. Since uniruledness is symplectic invariant [7, 13], we conclude that $Y$ is also uniruled for any symplectic deformation (Kähler threefold) $Y$ of $X$. Hence, $K_{Y}$ is not nef. However, below we will provide a more direct proof without using the abundance conjecture.

Lemma 4.7. If $\pi: \tilde{S} \rightarrow B$ is a ruled surface over a smooth curve $B$, then $\mathrm{GW}_{0,[C]} \neq 0$. 
Proof. First we note that $\operatorname{dim} \overline{\mathscr{M}}_{[C]} \geq-\left(K_{X}, C\right)=1$. If $\operatorname{dim} \overline{\mathscr{M}}_{[C]}>1$, then $\left(C_{t}\right)$ will fill up $X$ because $\operatorname{dim} \mathscr{M}_{[C]}(S)=1$ by hypothesis. Accordingly, $\mathrm{GW}_{0,[C]}(x) \neq 0$ where $x$ is the class of any point in $X$. If $\operatorname{dim} \overline{\mathscr{M}}_{[C]}=1$, then the deformation of $C$ is unobstructed; we can therefore conclude by Lemma 4.4.

From now on, we concentrate on the last remaining case in $(2 . b)$, that is the case where the normalization of $S$ is $\mathbb{P}^{2}$. Theorem 1.1 in this case will be a direct consequence of the following.

Proposition 4.8. In the case (2.b), if the normalization of $S$ is $\mathbb{P}^{2}$, then $X$ is uniruled.

Admitting Proposition 4.8 for the moment, we finish the proof of Theorem 1.1 as follows: since uniruledness is symplectic invariant [7, 13], we conclude that $Y$ is also uniruled so $K_{Y}$ is not nef.

Proof of Proposition 4.8. We will first prove the following lemma:

Lemma 4.9. $X$ is a fiber space over a smooth curve and $S$ is contracted to a point.

Proof. Assume that $H^{1}\left(X, \mathscr{O}_{X}\right)=0$. By considering the following short exact sequence:

$$
0 \longrightarrow H^{0}\left(X, \mathscr{O}_{X}\right) \longrightarrow H^{0}\left(X, \mathscr{O}_{X}(S)\right) \longrightarrow H^{0}\left(X, \mathscr{O}_{S}(S)\right) \longrightarrow 0, ;
$$

it is clear that $\operatorname{dim} H^{0}\left(X, \mathscr{O}_{X}(S)\right) \geq 2$. The morphism $X \rightarrow \mathbb{P}^{1}$ defined by the base-point-free linear system $\left|\mathscr{O}_{X}(S)\right|$ does the work.

If $H^{1}\left(X, \mathscr{O}_{X}\right) \neq 0$, then the Albanese map $\alpha: X \rightarrow \operatorname{Alb}(X)$ is nonconstant. If $\alpha$ is generically finite, then the pullback of a general holomorphic 3 -form is non-zero in $H^{0}\left(X, K_{X}\right)$, which contradicts the fact that $\kappa(X)<0$.

Now we assume that $\operatorname{dim} \operatorname{Im} \alpha \leq 2$. Let $\omega_{0}$ be a Kähler form on $\operatorname{Alb}(X)$ and $Q(\beta, \gamma):=\int_{X} \beta \wedge \gamma \wedge \omega$ be the intersection form on $H_{\mathbb{R}}^{1,1}(X):=H^{1,1}$ $(X, \mathbb{C}) \cap H^{2}(X, \mathbb{R})$ determined by $\omega$. The signature of $Q$ is $\left(1, \operatorname{dim} H_{\mathbb{R}}^{1,1}\right.$ $(X)-1)$ by Hodge index Theorem [17, Theorem 6.2.3]. Note that since there is no non-constant map from $\mathbb{P}^{2}=\tilde{S}$ to any torus, $\alpha$ contracts $S$ to a point, so $Q\left(\alpha^{*} \omega_{0},[S]\right)=0$. Since $\operatorname{dim} \operatorname{Im} \alpha \leq 2$, one has $Q\left(\alpha^{*} \omega_{0}, \alpha^{*} \omega_{0}\right) \geq 0$, it follows that $Q$ is negative on the orthogonal complement in $H_{\mathbb{R}}^{1,1}(X)$ of the line spanned by $\left[\alpha^{*} \omega_{0}\right]$. We then deduce from the fact that $Q\left(\alpha^{*} \omega_{0},[S]\right)=0$ and $\mathscr{O}_{S}(S)=\mathscr{O}_{S}($ so $Q([S],[S])=0)$ that $[S]$ is proportional to $\left[\alpha^{*} \omega_{0}\right]$. Hence, $\operatorname{dim} \operatorname{Im} \alpha=1$. Finally by [1, Proposition I.13.9], $\alpha(X)$ is smooth. 
By Lemma 4.9, $X$ is a fiber space over a smooth curve $\pi: X \rightarrow B$ such that $S$ is an irreducible component of a fiber $F$. Since $Q([S],[S])=$ $Q([S],[F])=0, S$ is in fact a connected component of $F$. Assume that $F$ is not connected. Given an irreducible component $S^{\prime}$ of $F$ disjoint with $S$, since $Q([S],[S])=0$ and $Q([F],[F])=0$, one would have $Q([S],[F]) \neq 0$ (otherwise $[S]$ would be proportional to $F$ again by Hodge index Theorem). Thus $Q\left([S],\left[S^{\prime}\right]\right) \neq 0$, which yields a contradiction. Hence, the fibers of $\pi$ are connected. In particular, we obtain $S \cdot C=0$

Now since $S$ is a Gorenstein surface, by [9, 3.34.1] (stated in the algebraic case, but the same proof works in the analytic case) one has $K_{\tilde{S}}=\nu^{*} K_{S}-E$ where $E$ is the pre-image of the non-normal locus of $S$ under $\nu$. Let $\tilde{C} \subset \tilde{S}$ be the strict transform of $C$. Recall that $K_{X} \cdot C=-1$ and $N_{S / X} \simeq \mathscr{O}_{S}(S)$, we obtain

$$
-3=\operatorname{deg}_{\tilde{C}}\left(K_{\tilde{S}}\right)=K_{X} \cdot C+S \cdot C-\tilde{E} \cdot \tilde{C}=-1-\tilde{E} \cdot \tilde{C}
$$

Hence, $\tilde{E} \cdot \tilde{C}=2$. Accordingly, $\nu^{*} K_{S}=\mathscr{O}_{\mathbb{P}^{2}}(-1)$, since $\tilde{C}$ is a line in $\tilde{S}$.

Therefore, one has $H^{0}\left(S, n K_{S}\right)=0$ for all $n>0$, so $\kappa(S)=-\infty$. The same result extends by semi-continuity to all fibers of points lie in a Zariski neighborhood of $\pi(S)$, i.e., there is a non-empty Zariski open $U \subset B$ such that $S_{t}$ is smooth and $\kappa\left(S_{t}\right)=-\infty$ for all $t \in U$ where $S_{t}:=\pi^{-1}(t)$. These $S_{t}$ are all uniruled, so $X$ is also uniruled.

Remark 4.10. We could have concluded the proof of Proposition 4.8 by the result of Peternell [10] mentioned in Remark 4.6 once we know that the Albanese map $\alpha$ is not generically finite. Indeed, since $X \rightarrow B$ is fibered over a base $B$ of dimension strictly between 0 and $\operatorname{dim}(X), X$ is not a simple manifold. Since $X$ has negative Kodaira dimension, $X$ is uniruled.

\section{Acknowledgments}

I heartily thank my advisor Claire Voisin for introducing me to this topic and for sharing with me her enlightening remarks.

\section{References}

[1] W. Barth, K. Hulek, C. Peters and A.V.D. Ven, Compact complex surfaces, 4, Ergebnisse der Mathematik und ihrer Grenzgebiete, Springer, 2nd ed., 2004. 
[2] F. Campana, A. Höring and T. Peternell, Abundance for Kähler threefolds, 2014, arXiv:1403.3175.

[3] F. Campana and T. Peternell, Towards a Mori theory on compact Kähler threefolds I, Math. Nachr. 187 (1997), 29-59.

[4] W. Fulton and R. Pandharipande, Notes on stable maps and quantum cohomology, in Algebraic geometry — Santa Cruz 1995, Proc. Symp. Pure Mathematics 62 (1997), 45-96.

[5] K. Hori, S. Katz, A. Klemm, R. Pandharipande, R. Thomas, C. Vafa, R. Vakil and E. Zaslow, Mirror symmetry, Clay Math. Monographs, 1, Amer. Math. Soc. and Clay Inst., 2003.

[6] A. Höring and T. Peternell, Minimal models for Kähler threefolds, 2013, arXiv: 1304.4013.

[7] J. Kollár, Low degree polynomial equations: arithmetic, geometry and topology, in European Congress of Mathematics, Vol. I, (Budapest, 1996), Vol. 168 of Progress in Mathematics, 255-288, Birkhäuser, 1998.

[8] J. Li and G. Tian, Comparison of algebraic and symplectic GromovWitten invariants, Asian J. Math. 3(3) (1999), 689-728.

[9] S. Mori, Threefolds whose canonical bundles are not numerically effective, Ann. Math. 116 (1982), 133-176.

[10] T. Peternell, Towards a Mori theory on compact Kähler threefolds III, Bull. Soc. Math. France 129(3) (2001), 339-356.

[11] Y. Ruan, Symplectic topology and extremal rays, Geom. Funct. Anal. 3(4) (1993), 395-430.

[12] - Symplectic topology on algebraic 3-folds, J. Diff. Geometry 39 (1994) 215-227.

[13] - Virtual neighborhood and pseudo-holomorphic curves, in Proc. of 6th Gökova Geometry-Topology Conf. 23 (1999), 161-231.

[14] B. Siebert, Algebraic and symplectic Gromov-Witten invariants coincide, Ann. Inst. Fourier 49 (1999), 1743-1795.

[15] Z. Tian, Symplectic geometry and rationally connected 4-folds, J. Reine Angew. Math. 2015 (698) (2015), 221-244.

[16] - Symplectic geometry of rationally connected threefolds, Duke Math. J. 161(5) (2012), 803-843. 
[17] C. Voisin, 'Théorie de Hodge et géométrie algébrique complexe', Cours Spécialisés, Société Mathématique de France, 10 (2002).

[18] —, Rationally connected 3-folds and symplectic geometry, in 'Géométrie différentielle, physique mathématique, mathématiques et société II', Astérisque 322 (2008), 1-21.

CMls, École Polytechnique

École Polytechnique

91128 PALAISEAU CEDEX

FRANCE

E-mail address: hsueh-yung.lin@polytechnique.edu

ReCeIVEd March 25, 2013 